\title{
Intraoperative Magnetic Resonance Imaging with Evoked Potential Monitoring: Tailored Method of Scalp Corkscrew Electrode Placement to Reduce Image Artifacts
}

\author{
Nitin Manohar ${ }^{1}$ Astha Palan ${ }^{1}$ \\ Dhritiman Chakrabarti ${ }^{4}$ \\ ${ }^{1}$ Department of Neuroanesthesiology, Yashoda Hospitals, \\ Secunderabad, Telengana, India \\ 2Department of Anaesthesiology, Rainbow Children's Hospital, \\ Hyderabad, Telengana, India \\ ${ }^{3}$ Department of Neuroanaesthesia, Apollo Hospitals, Bengaluru, \\ Karnataka, India \\ ${ }^{4}$ Department of Neuroanaesthesia and Neurocritical Care, National \\ Institute of Mental Health and Neuro Sciences, Bengaluru, \\ Karnataka, India
}

J Neuroanaesthesiol Crit Care 2019;6:45-47

Intraoperative magnetic resonance imaging (IMRI) is an essential tool in the age of image-guided neurosurgery for distinguishing between abnormal and normal brain tissues, minimizing tumor residue, and guiding accurate tumor resection in both brain and spine procedures. It involves imaging following surgery and prior to definitive closure of surgical site wound and extubation.

Intraoperative neuromonitoring (IONM) forms a crucial part of neurosurgery to ensure safe resection of tumors and prevent damage to critical structures. Cases wherein IONM is ongoing in the form of evoked potential monitoring necessitate bipolar corkscrew electrodes placement on the scalp. These electrodes are generally made of stainless steel that is certified for IONM, but not strictly "MRI conditional." The safe use of these electrodes during IMRI has been demonstrated in invitro studies, ${ }^{1}$ although the possibility of excessive heating by the radiofrequency field cannot be excluded, and hence the risk-benefit ratio must guide their usage. They possess ferromagnetic properties, giving rise to multiple artifacts on the MRI scans when left in situ ${ }^{1}$ ( - Fig. 1A). If removed, the electrodes cannot be replaced as it compromises the sterility of the surgical field and the rest of the surgery or residual tumor resection will have to proceed without IONM. This predicament essentially affects the cases requiring intraoperative brain imaging.

The placement of scalp corkscrew electrodes is based on the international 10-20 system. Somatosensory evoked potential (SSEP) monitoring requires placement of two or three corkscrew electrodes for upper limb (C3', C4', and Fz) and lower limb ( $\mathrm{Cz}$ and $\mathrm{Fz} / \mathrm{Fpz}$ ) monitoring. Similarly, transcranial
Address for correspondence Deepti Srinivas, MD, PDF, Department of Neuroanaesthesia, Apollo Hospitals, Bannerghatta Road, Bengaluru 560076, Karnataka, India (e-mail: bsdeepti@gmail.com).

motor evoked potential (MEP) monitoring requires two electrodes for upper limb (C3, C4) and lower limb $(\mathrm{C} 3, \mathrm{C} 4$, or $\mathrm{Cz}, \mathrm{Fz} / \mathrm{Fpz}$ ). Hence when SSEP and MEP are used in combination, a total of four or five corkscrew electrodes need to be placed.

We describe a tailored method of corkscrew electrode placement on the scalp, which reduces its number and thus subsequently decreases the artifacts on MRI scans. The IMRI setup at our center is a 3-Tesla "nearby operating room" type with the Siemens Skyra magnet located adjacent to the standard operating theater. Ethical clearance was not required for this procedure and hence not obtained. Written informed consent was obtained from the patient prior to writing this report.

Our technique involves utilizing the same set of corkscrew electrodes for SSEP and MEP monitoring by simply changing their connections at the recorder/stimulator box. For instance, a case requiring upper limb SSEP + all four limbs MEP necessitates placement of five electrodes: C3', C4', and Fz for SSEP and C3 and C4 for MEP ( - Fig. 2A). Instead, we placed three electrodes: $\mathrm{C}^{\prime}, \mathrm{C4}^{\prime}$, and $\mathrm{Fz} . \mathrm{C3}^{\prime}$ and $\mathrm{C4}^{\prime}$ were connected to the recorder box for continuous SSEP monitoring (-Fig. 2B). The same can be connected to the stimulator box for monitoring intermittent MEP. The voltage strength we used for stimulation was within the standard recommended range (150-200 V). We utilized this method in 15 patients, and none of them had any complications (intraoperative seizure, scalp burns, tongue bite). We were successfully able to obtain satisfactory waveforms for muscle MEP ( - Fig. 3 ) in all despite the electrodes placed at received

May 28, 2018

accepted after revision

September 12, 2018

published online

January 25, 2019
DOI https://doi.org/

10.1055/s-0038-1675890

ISSN 2348-0548.
Copyright $\odot 2019$ Indian Society of Neuroanaesthesiology and Critical Care
License terms

() (1) $\Theta \circledast$ 


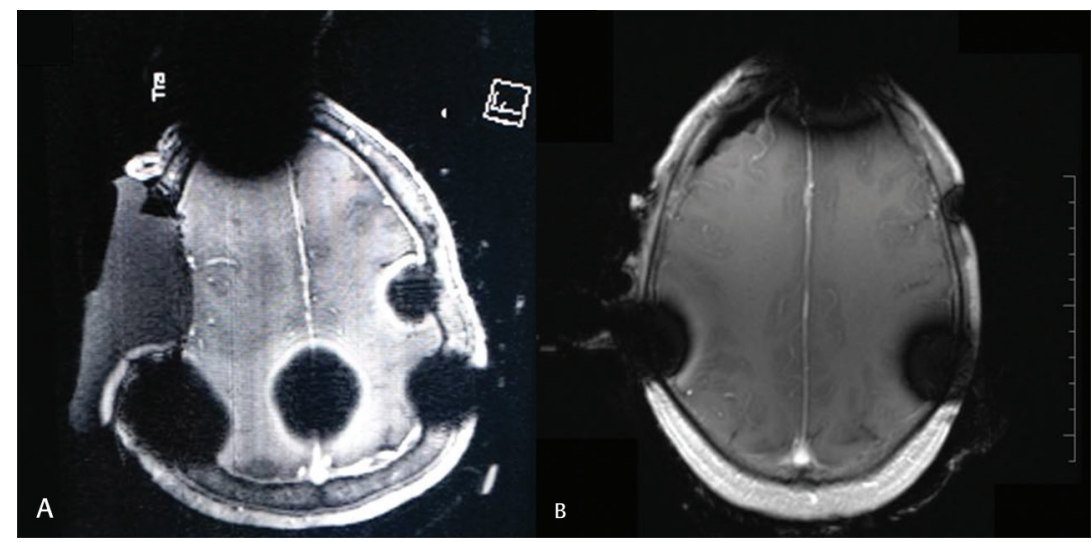

Fig. 1 (A) Axial section of IMRI of the brain showing artifacts due to corkscrew electrode placement. (B) Axial section of IMRI of the brain showing reduction in artifacts following alternate method of electrode placement (images not from the same patient). IMRI, intraoperative magnetic resonance imaging.

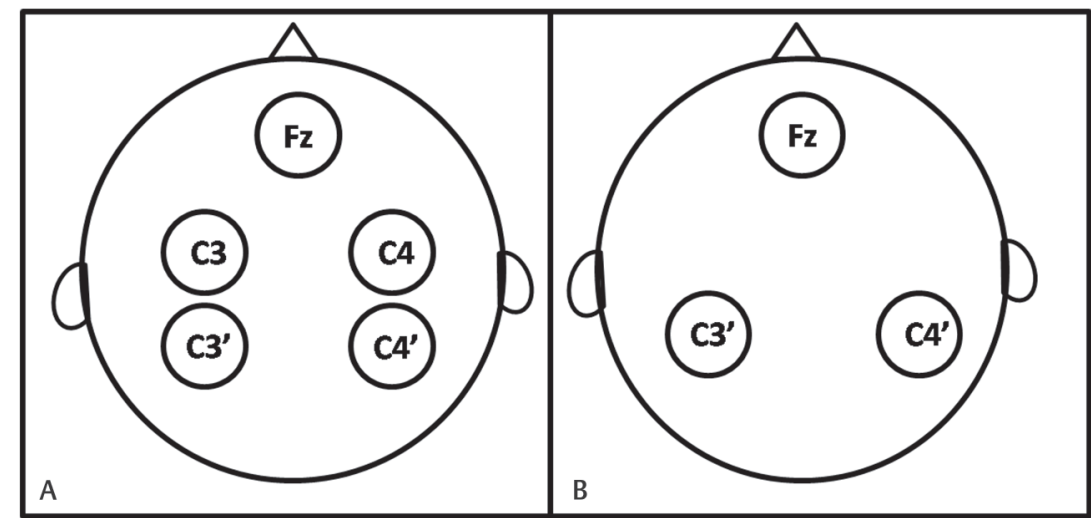

Fig. 2 (A) Conventional method of corkscrew electrode placement (as per the international 10-20 system) for bilateral SSEP + MEP involving five electrodes-a pictorial representation. (B) Modified method using three electrodes.

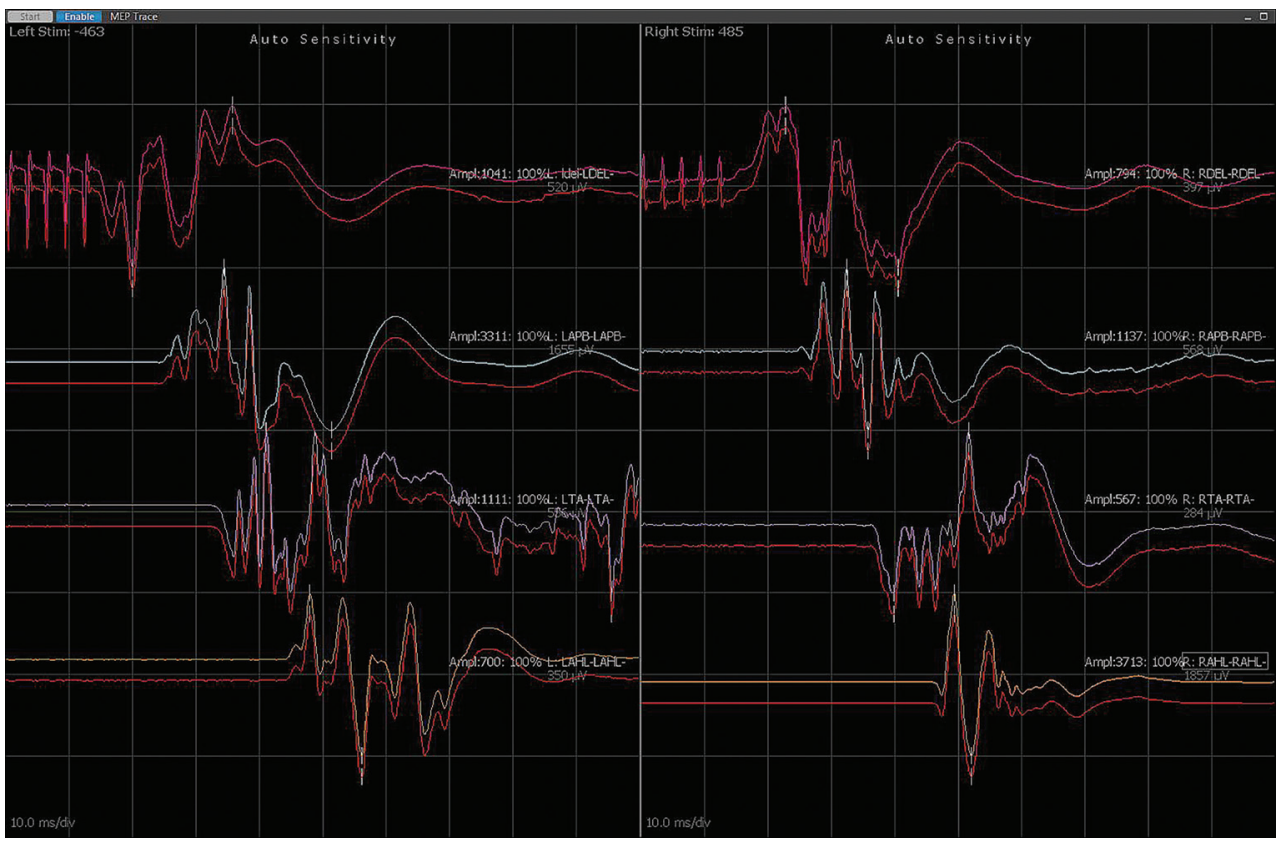

Fig. 3 The MEP waveform figure obtained when using modified method. MEP; magnetic resonance imaging. 
the alternative location, and the IMRI scans showed fewer artifacts $(-$ Fig. 1B). Furthermore, the reduction in number of corkscrew electrodes implies fewer sites of its traumatic insertion on the scalp.

Combined SSEP and MEP monitoring has been seen to be beneficial in craniotomy for insular glioma, tumors near vascular territories (e.g., near anterior cerebral artery), frontal tumor near skull base, anterior circulation aneurysm repair surgery, cavernoma resections, and as compared with use of either one modality in isolation., ${ }^{2,3}$ Using our alternative approach of corkscrew electrode placement serves the dual purpose of reducing artifacts on IMRI scans as well as the number of screw insertions on the scalp without interfering with the IONM aspect. However, this method of alternative placement may not be able to stimulate the motor cortex in all cases, increasing the incidence of false negativity; hence, electrode placement in such cases may need to be individualized. Moreover, before using it as a standard method, results in terms of image and waveform quality should be further evaluated.

\section{Funding}

None.

\section{Conflict of Interest}

None declared.

\section{References}

1 Sarnthein J, Lüchinger R, Piccirelli M, Regli L, Bozinov O. Prevalence of complications in intraoperative magnetic resonance imaging combined with neurophysiologic monitoring. World Neurosurg 2016;93:168-174

2 Weinzierl MR, Reinacher P, Gilsbach JM, Rohde V. Combined motor and somatosensory evoked potentials for intraoperative monitoring: intra- and postoperative data in a series of 69 operations. Neurosurg Rev 2007;30(2):109-116, discussion 116

3 Choi I, Hyun SJ, Kang JK, Rhim SC. Combined muscle motor and somatosensory evoked potentials for intramedullary spinal cord tumour surgery. Yonsei Med J 2014;55(4):1063-1071 